La dimension politique de l'éducation relative à l'environnement

\title{
Changements climatiques à l'école: Pour une éducation sociopolitique aux sciences et à l'environnement
}

\section{Virginie Albe}

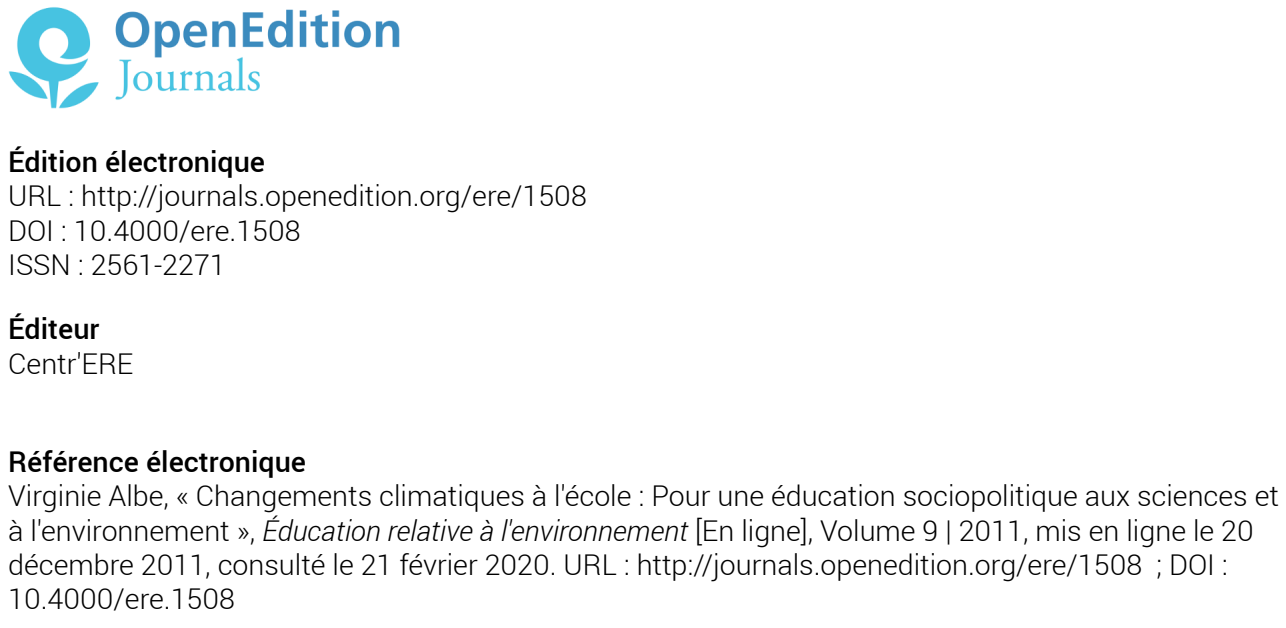

Ce document a été généré automatiquement le 21 février 2020. 


\title{
Changements climatiques à l'école : Pour une éducation sociopolitique aux sciences et à l'environnement
}

\author{
Virginie Albe
}

1 Depuis plusieurs années, des recherches ont été développées sur le traitement didactique de questions dites " socialement vives", en sciences économiques et sociales d'abord, puis en sciences de la matière et du vivant (Legardez et Simonneaux, 2006). Selon les chercheurs, les questions scientifiques considérées socialement vives rejoignent des problématiques en lien avec l'environnement et/ou la santé, qu'il s'agisse, par exemple, des controverses sur l'énergie et les changements climatiques, les technologies de la téléphonie mobile, les organismes génétiquement modifiés ou les nanotechnologies. Les développements technoscientifiques soulèvent souvent des débats au sein des communautés savantes et entre elles; ils font également l'objet de débats en société et d'expertises scientifiques (parfois aussi juridiques) contradictoires. Les acteurs de la sphère politique sont également amenés à prendre des décisions à leur propos, en contexte d'incertitude et en présence d'une variété d'expertises. Leur double vivacité, scientifique et sociale, a conduit à les envisager du point de vue de la recherche en didactique des disciplines comme des questions scientifiques socialement vives (Legardez et Alpe, 2001), des controverses socioscientifiques (Albe, 2007) ou des socioscientific issues, dans les recherches en langue anglaise (Sadler, 2004, 2009). Une analyse de type épistémologique et sociologique, visant à identifier les savoirs et les acteurs de telles controverses socioscientifiques, met en évidence leur nature politique, au point que l'on peut considérer ces questions comme avant tout "politiquement vives ». Par ailleurs, des recherches empiriques sur le traitement de telles controverses socioscientifiques en classe ont également souligné que les élèves non seulement sont aptes à appréhender leur dimension politique, mais l'intègrent aussi spontanément dans leurs discussions, même si le dispositif didactique conçu ne l'envisageait pas. C'est ce que nous aborderons dans cet article, en discutant les résultats d'une recherche portant sur l'étude des controverses sur les changements climatiques en milieu scolaire français, plus précisément au niveau secondaire. Mais d'abord, nous présenterons le 
champ des recherches sur les controverses socioscientifiques et leurs enjeux socioéducatifs. Enfin, nous examinerons les débats et les critiques, dans le champ de la recherche et de l'intervention éducative, portant sur la prise en charge à l'école, et en particulier en classe de sciences, de la dimension politique de telles controverses.

\section{Enjeux socioéducatifs de l'enseignement de controverses socioscientifiques}

2 L'émergence de recherches en didactique sur des questions scientifiques dites «socialement vives» à la fin des années 1990 est ancrée dans un contexte socioéducatif et scientifique. L'accent y est mis sur la promotion d'un enseignement des sciences qui contribue à une éducation à la citoyenneté et à la responsabilité, sur le développement des "éducations à » ... (la santé, la sexualité, l'environnement et le développement durable, etc.). Dans la recherche en éducation aux sciences, s'affirme également un intérêt accru pour la culture scientifique et technique pour tous, et pour l'argumentation en classe de sciences.

3 Par ailleurs, le courant anglophone des socioscientific issues peut être situé dans la lignée des recherches en éducation aux sciences dites "sciences-technologies-sociétés", développées depuis les années 1970 en réaction à une vision des sciences et de l'enseignement scientifique essentiellement positiviste. Il rejoint également à plusieurs égards les recherches en éducation à l'environnement : l'enseignement des sciences est une entreprise qui dépasse le cadre scolaire et permet aux étudiants de développer une connaissance profonde $\mathrm{du}$ monde dans lequel ils vivent. Avec une telle finalité socioéducative, l'enseignement des sciences doit prendre en compte explicitement des préoccupations sociales.

4 L'enseignement de questions scientifiques socialement vives ou de controverses socioscientifiques s'inscrit ainsi dans une perspective d'éducation aux sciences plus citoyenne et vise une démocratisation des technosciences pour tous. Il s'agit de permettre aux citoyens de comprendre et de participer aux débats de nos sociétés modernes sur des problèmes socioscientifiques et technoscientifiques toujours plus complexes (Driver et al., 1996 ; Kolstø, 2001 ; Bader, 2003 ; Sadler, 2004).

Des recherches se sont ainsi développées dans le champ des didactiques disciplinaires sur des questions socialement vives en sciences, en sciences économiques, sociales et de gestion, en histoire et en géographie. Leur théorisation est naissante. Le terme de " questions socialement vives » est utilisé par Legardez $(1999$, p. 49) pour désigner des objets de savoirs économiques, sociaux et de gestion dont la transposition didactique peut s'avérer particulièrement délicate du fait de leur vivacité sociale. Legardez (2006, p. 21-22) définit ensuite une question triplement socialement vive, lorsqu'elle est « vive » à la fois dans la société, dans les savoirs de référence et en classe. Il ne s'agit pas toujours de "questions d'actualité » et, dans l'enseignement, ces objets ne se présentent que rarement sous une forme explicite de questionnement (Legardez, 2006, p. 22). Leurs degrés de vivacité sont variables : des questions peuvent être "émergentes» (ex. : les OGM ou la mondialisation), d'autres "latentes» (ex. : le racisme, l'entreprise) ou " résurgentes" (ex. : l'énergie), comme l'indique Legardez (2006), ou encore "oubliées » (ex. : le saturnisme ou le paludisme), tel que le soulignent Larochelle et Désautels (2006, p. 75). 
6 La formation du citoyen est la finalité généralement exprimée dans toutes les recherches menées dans le courant des questions scientifiques dites socialement vives. Toutefois, si toutes ces recherches se réfèrent à une éducation citoyenne, une analyse des travaux montre une grande diversité dans les approches. À des fins analytiques, deux options extrêmes peuvent être identifiées (Albe, 2009). Il peut s'agir de concevoir les questions socioscientifiques comme moyen de favoriser l'apprentissage de savoirs scientifiques. Une autre approche comporte une finalité émancipatoire au traitement de questions socioscientifiques. Il s'agit, si nous traduisons le terme anglais " empowerment », souvent utilisé et illustratif de cette tendance, de donner le pouvoir aux élèves pour participer aux débats publics, aux prises de décisions en matière technoscientifique et à la configuration de la société.

\section{Un traitement en classe des controverses sur les changements climatiques}

7 Nous présentons brièvement ici une expérimentation en classe d'enseignement de controverses sur les changements climatiques (Gombert, 2008 ; Albe et Gombert, 2010) qui a pour enjeu éducatif de permettre aux élèves : de développer une compréhension avertie des pratiques scientifiques et de l'imbrication des questions de sciences, de techniques et de société ; d'opter pour un usage critique et raisonné de l'expertise ; de participer aux débats publics. Pour ce faire, nous avons conçu une séquence d'enseignement interdisciplinaire sur les changements climatiques avec des enseignants de philosophie, de sciences physiques et de biologie-écologie pour une classe de terminale technologique de l'enseignement agricole.

La nature politique de l'objet proposé en discussion aux élèves (les changements climatiques), sa couverture médiatique et son caractère vif en société en font une controverse socioscientifique qui mobilise et divise des groupes sociaux. En conséquence, notre équipe d'enseignants et de chercheures a adopté une attitude de prudence quant à la mise en œuvre de débats en classe. Nous avons voulu éviter de confiner les discussions des élèves à des affrontements stériles sur le plan de l'apprentissage et générateurs de violence ou de mimer en classe un simulacre de débat public inspiré des formes médiatiques de débat. Par ailleurs, la recension d'écrits que nous avions préalablement menée nous avait permis d'identifier, dans les résultats des recherches mettant en œuvre en classe des débats sur des questions scientifiques socialement vives, que les élèves pouvaient être amenés à argumenter pour "gagner " plutôt qu'à s'engager dans l'étude des controverses (Albe, 2005). En outre, des approches issues de la linguistique ou de la sociolinguistique, utilisées pour comprendre les pratiques discursives d'élèves en travail autonome en petits groupes, mettent l'accent sur des difficultés à l'écoute et au dialogue (Dawes, 2004 ; Mercer, 1996). Des discours modélisés comme cumulatifs où chacun s'exprime dans le groupe sans qu'une interaction se développe, des discours d'oppositions ou d'affrontements sont fréquents. Plus rares sont les discours d'exploration où chacun contribue à une élaboration orale collective (Mercer, 1997). L'équipe interdisciplinaire a alors fait le choix d'intégrer dans la séquence d'enseignement une initiation à la communication non violente (CNV) animée par une intervenante spécialisée. La communication non violente est d'abord une méthode de dialogue et d'échanges dans le respect de nousmêmes, de nos besoins et de ceux de nos interlocuteurs. Ce processus de 
communication initié dans les années 1970 par le psychologue Rosenberg (1999) s'appuie sur l'idée de besoins humains partagés par chacun et met l'empathie au centre de la communication. En référence au mouvement de Gandhi, la non-violence consiste ici à communiquer avec l'autre sans lui nuire et à développer des échanges qui se substituent au conflit. Les quatre étapes d'un processus CNV sont les suivantes : décrire la situation en termes d'observation partageable (il s'agit en particulier de distinguer les observations de jugements ou d'évaluations), exprimer ses sentiments (il s'agit en particulier de distinguer nos émotions et la perception que nous nous construisons de l'autre), exprimer ses besoins et demander les actions que l'on souhaite.

9 Les quinze élèves et les deux chercheures ${ }^{1}$ ont participé à deux séances de formation à la communication non violente (séances 1 et 2 des cinq que comporte la séquence pédagogique, respectivement d'une durée de $7 \mathrm{~h} 30$ et $3 \mathrm{~h}$ ) avec mises en situation de débats, analyses et formalisation de la communication sur des conflits du quotidien ou liés à des développements technoscientifiques (comme celui de la patate génétiquement modifiée ou ceux liés aux recherches sur le paludisme ou le sida, par exemple). À ce propos, soulignons ici que le choix des thèmes à débattre a fait l'objet d'une élaboration collective et que les élèves ont mis en avant la nécessité de choisir des situations authentiques et/ou comportant, à leurs yeux, une dimension politique. Ont été écartées par exemple les questions relatives aux recommandations de pratique régulière d'un sport, aux marques de vêtement ou au tabagisme. À l'issue de ces 2 séances, l'équipe a envisagé le débat sur les changements climatiques comme espace d'apprentissage, d'exploration des controverses en vue d'une résolution citoyenne dans un cadre démocratique et comme alternative à la violence des échanges entre élèves.

Lors de la séance $3(2 \mathrm{~h})$, le film "Une vérité qui dérange » avec $\mathrm{Al} \mathrm{Gore}{ }^{2}$ a fait l'objet d'une projection et d'une analyse critique pilotée par l'enseignant de philosophie, avec la participation de l'enseignant de biologie-écologie et des chercheures. L'analyse du film correspond à une pratique coutumière dans la classe de philosophie de cet enseignant $^{3}$. Il s'agit de caractériser la forme et d'examiner comment celle-ci est mise au service du fond. Les élèves ont évoqué la question de la mise en scène dramatique (évocation de l'accident du fils de Al Gore, cancer de sa meilleure amie d'enfance, souvenir de vie familiale rurale...), les rôles multiples de Al Gore non explicités (homme politique, acteur, individu isolé qui se bat pour une cause morale, spécialiste des questions d'environnement, utilisateur de gros véhicules $4 \times 4$...), les formes multiples $\mathrm{du}$ film (documentaire scientifique, plaidoyer écologique, biographie ou autobiographie...). Les élèves ont également discuté de l'importance d'être sensible aux questions d'environnement et du rôle bénéfique du film de ce point de vue là.

11 C'est au cours de la quatrième séance que les élèves ont été mis en situation de débattre, en adoptant le modèle d'une conférence de citoyens autour de la question controversée du climat. Dans un premier temps, une chercheure a procédé à la lecture du document "présentation de la situation débat». Celui-ci a été élaboré spécifiquement pour cette séance, en tenant compte des préoccupations de l'équipe issues de l'analyse des séances précédentes. De cette analyse réalisée en équipe est ressorti qu'il fallait créer une situation non fictive et impliquer la dimension politique. Il est question, dans la première partie de ce document, de présenter la controverse du changement climatique en lien avec le film «Une vérité qui dérange " (visionné à la séance précédente) et de situer ce film dans l'actualité du Prix Nobel de la paix décerné en octobre 2007 à Al Gore ainsi qu'aux experts du Groupe intergouvernemental 
d'experts sur l'évolution du climat (GIEC). Dans la seconde partie, on peut lire que le film s'appuie sur le discours du GIEC pour insister sur l'urgence de réduire les émissions de gaz à effet de serre et que, dans cette perspective, la position du chef de l'État français en matière de politique énergétique internationale est de développer l'énergie nucléaire et de vendre des centrales nucléaires aux pays émergents, futurs grands consommateurs d'énergie. La France justifie cette politique par le fait que le nucléaire ne produit pas de $\mathrm{CO} 2$ (un des gaz à effet de serre). La dernière partie du document présente le jeu de rôles basé sur un modèle de conférence de citoyens. Il est question d'attribuer aux élèves des rôles bien définis en les leur expliquant. La situation de débat telle que décrite dans le document est la suivante :

Vous êtes des citoyens français informés sur les controverses scientifiques existantes en matière de climat et chargés de donner quatre recommandations pour les politiciens en matière de choix énergétique pour la France et/ou pour les pays émergents dans les années futures. Pour élaborer ces quatre recommandations, nous allons recréer une situation de débat prise sur un modèle existant de conférences de citoyens destinées à fournir un avis aux politiques.

Les rôles que nous avons conçus pour les élèves sont les suivants :

- Quatre experts répartis en deux équipes présentent l'un après l'autre au groupe de citoyens deux thèses opposées sur les changements climatiques à partir des documents fournis ;

- Sept citoyens ${ }^{4}$ questionnent ensuite les experts puis débattent des quatre recommandations à fournir aux décideurs politiques en matière de politique énergétique. Ils doivent spécifier sur quelle expertise s'appuie leur choix. À tout moment, lors de leurs discussions, les citoyens peuvent questionner les experts ;

- Deux modérateurs en communication non violente: à tout moment, lors de leurs discussions, les citoyens peuvent solliciter ces modérateurs ;

- Deux rapporteurs chargés de prendre en note toutes les discussions pour en rendre compte lors d'une phase de discussion sur la situation de débat.

Chaque élève s'est vu attribuer un rôle qui avait fait l'objet d'un choix concerté entre les chercheures et l'enseignant de philosophie. Ce choix d'imposer le rôle à jouer aux élèves relève de deux raisons essentielles : l'une en lien avec la gestion du temps (nous ne voulions pas que les élèves perdent du temps à choisir leur rôle) et l'autre en lien avec l'idée pour nous de favoriser leur engagement dans le débat (nous avons tenu compte de leurs implications dans les séances précédentes, mais aussi des facteurs relationnels et interpersonnels; nous voulions éviter que les élèves choisissent les rôles à jouer selon des critères interpersonnels).

Une fois les rôles attribués, les élèves ont été invités à se regrouper pour prendre connaissance du travail qui leur serait demandé en fonction du rôle à jouer. Des documents élaborés pour la séance et relatifs aux différents groupes (citoyens, experts, modérateurs $\mathrm{CNV}$ et rapporteurs) ont été distribués et commentés par les chercheures dans chacun des groupes. Une fois cette phase relative aux consignes propres à chacun des groupes terminée, les élèves experts ont été appelés à exposer à tour de rôle leurs thèses scientifiques respectives. Pour préparer leur exposé, chaque groupe d'experts avait à disposition un document spécifiquement élaboré pour la situation, présentant les thèses de différents scientifiques et groupes d'experts bien identifiés, en respectant les critères de référencement pratiqués dans la communauté scientifique. Il nous paraissait en effet important, au vu de notre positionnement épistémologique, de mettre à disposition des élèves une vision socialisée des savoirs scientifiques. En ce qui concerne la présentation des éléments de controverses sur le réchauffement 
climatique, l'équipe s'est appuyée sur l'analyse épistémologique et sociale des controverses de Albe (2008). Les chercheures ont élaboré les documents de travail « en face à face ", de telle sorte que les arguments opposés puissent être facilement identifiés par les élèves et afin de respecter l'équilibre idéologique des thèses en présence et la forme des documents.

Les exposés d'experts terminés, les élèves ont été invités à débattre pendant quinze minutes selon une disposition dans la classe élaborée sur le modèle de conférences de citoyens. Les échanges ayant eu lieu au cours de ce débat entre élèves jouant le rôle de citoyens, experts, modérateurs $\mathrm{CNV}$ et rapporteurs ont été intégralement enregistrés par vidéo et audio, puis intégralement retranscrits pour analyses ultérieures. Le débat terminé, les rapporteurs de séance ont lu les propositions écrites du groupe des citoyens en matière de politique énergétique pour la France et/ou les pays émergents. Un temps de retour sur la situation de débat au regard de la communication non violente a alors été mené par les modérateurs $\mathrm{CNV}$ qui rendaient compte de leurs observations pendant le débat. Les élèves ont ensuite été invités à compléter ou à relire leurs fiches pour les remettre ensuite aux chercheures. Cette quatrième séance s'est terminée par une conclusion élaborée et énoncée par les chercheures et portant sur l'annonce des deux niveaux d'analyse et de discussion des séances à être abordés dans la séance cinq (éléments de la recherche sur la CNV et réflexion du rôle du citoyen quant aux discours scientifiques et politiques). Au cours de la cinquième et dernière séance de cette séquence, nous avons engagé avec les élèves (à partir d'extraits de transcriptions des échanges ayant eu lieu au cours du débat précédent) des réflexions sur des éléments de la recherche que nous avions menée avec eux, sur la $\mathrm{CNV}$, ainsi que sur le rôle du citoyen quant aux discours scientifiques et politiques. Ce moment a été une occasion pour les chercheures de valider les analyses et d'aborder l'aspect éthique du travail de recherche effectué, en montrant aux élèves ce qui avait été fait à partir des enregistrements sonores, de la vidéo et des documents recueillis pendant les séances. Il s'est aussi agi pour nous de montrer aux élèves ce que nous avions pu faire grâce à eux. Un temps d'analyse collective où " tout est possible » - dans le sens d'une discussion ouverte - a terminé la séance.

Les transcriptions des discussions des élèves, organisées selon le modèle des conférences de citoyens, ont fait l'objet de plusieurs analyses, visant à identifier les savoirs mobilisés par les élèves lors du débat. Ces analyses se sont inspirées du prisme des outils de communication non violente développés par Rosenberg (1999), de l'approche de la psychologie discursive anglaise (Edwards, 1997 ; Potter, 1997), afin de documenter les jeux rhétoriques, et de la dimension épistémologique du modèle d'une écologie des controverses socioscientifiques (Albe, 2007).

\section{Des discussions non-violentes entre élèves}

17 La transcription des échanges au cours du débat a permis d'identifier que les élèves chargés d'un rôle de modérateurs CNV sont intervenus à six reprises et qu'un total de 252 interventions ont composé le débat. L'une de ces six interventions mobilise la notion de jugement, telle qu'elle apparaît dans le processus CNV défini par Rosenberg (1999). CNV1 $1^{5}$ s'adresse à un expert opposé à la thèse du GIEC, réagissant à ces propos :

I205 E1 : ... [E1 prend son document d'expertise et lit] « des scientifiques ont par

ailleurs, indiqué l'absence de consensus scientifique sur le réchauffement 
climatique ». Bon peut-être que vous ne me comprenez pas, vous êtes l'un des

péqueux lambda.

transcription du débat, on observe que leurs affirmations, à l'écrit, sont en cohérence avec la situation vécue. Nous observons que sur les trois réponses des modérateurs CNV dans la fiche post-débat, deux d'entre elles ont fait l'objet d'interventions d'un des modérateurs $\mathrm{CNV}$ au cours du débat. L'idée de "jugement » associée à l'emploi du mot "péqueux ", tel que les modérateurs $\mathrm{CNV}$ en ont rendu compte dans la fiche postdébat, fait l'objet de l'intervention I209 réalisée par CNV1 :

I209 CNV1: Hé hé Monsieur [CNV1 interpelle E1] il y a un petit jugement sur les citoyens.

21 On peut faire la même remarque à propos de la coupure de parole entre les deux groupes d'experts signalée en réponse à la deuxième consigne dans la fiche post-débat. L'élève mentionne l'intervention I133 de la part du modérateur CNV2 :

I133 CNV2 : on ne coupe pas la parole.

Nous constatons ainsi une cohérence entre les écrits des élèves en post-débat et leurs interventions au cours de celui-ci. À l'écrit, les élèves ont mentionné un élément supplémentaire (référence aux propos ridiculisant d'un élève citoyen vis-à-vis d'un expert) qui n'a pas fait l'objet d'intervention orale. Ce résultat nous conforte dans l'idée du rôle complémentaire d'une activité mobilisant l'écrit à l'issue du débat pour que les élèves s'engagent dans une réflexion sur la séance, telle que vécue.

\section{Les élèves et la construction des expertises scientifiques ${ }^{6}$}

\section{Une expertise sans intérêt financier}

Dans de nombreux cas, les deux groupes d'experts se voient attribuer des intérêts d'ordre financier dans le but d'affaiblir leur position. En réaction à l'attribution d'intérêt, l'expert E1 réfute à plusieurs reprises le lien entre financement et résultats de recherches, en avançant l'idée qu'il est indépendant ou bien qu'il travaille " totalement gratuitement» (I178). De son côté, l'expert du GIEC, EG1, s'il reconnaît avoir été sélectionné par un gouvernement (comme cela est écrit dans le document d'expertise à

Éducation relative à l'environnement, Volume 9| 2011 
la définition du GIEC), se défend de tout lien financier en déclarant : « Mais ils ne nous donnent pas de l'argent » (I158).

«démontrer» qu'il existe bien une influence du politicien sur le scientifique (en l'occurrence ici le GIEC). L'expert E1 déclare : « Un gouvernement c'est politique et les politiques font dire aux scientifiques ce qu'ils veulent; ils utilisent ceux qu'ils veulent ». Le lien sciences-politique est donc très directement discuté par une partie des élèves, avec l'idée pour certains d'une récupération du scientifique et de ses travaux à des fins politiques. Dans cet esprit, la question des intérêts politiques que pourrait avoir $\mathrm{Al}$ Gore à véhiculer les thèses du GIEC, dans le film «Une vérité qui dérange » visionné en classe, est rapportée par l'expert E2 à deux reprises (I44, I45), en réponse à la question posée par l'élève citoyen C1 (I42) sur l'enjeu politique que pourrait susciter la question environnementale. Peu après, E2 renforce l'idée de l'absence de lien entre les scientifiques et le politique en déclarant ceci: «On est censés, en tant que scientifique, ne pas avoir d'avis scientifique, ... politique » (I52). Il est intéressant de noter que E2 hésite dans cette intervention et se trompe dans un premier temps entre "d'avis scientifique» et «d'avis politique» et emploie l'expression «on est censés» qui pourrait mettre en doute ses propres propos. Par ailleurs, dans son intervention (I159), l'expert E1 défend l'idée que les pratiques politiciennes de "récupération des scientifiques qui les arrangent » relèvent d'un régime dictatorial. Pointe alors ici la question des relations entre scientifiques et systèmes politiques, l'usage politique de l'expertise pouvant éloigner du système démocratique.

L'analyse des jeux rhétoriques relevant de l'attribution d'intérêts dans le but de miner un résultat scientifique ou une position d'expert scientifique montre que ces procédés sont largement mobilisés par les élèves au cours du débat. Les élèves soulèvent la question des financements des recherches, des liens entre politique et sciences et du caractère désintéressé du travail scientifique. Il nous paraît important de noter que si des orientations politiques de la part de scientifiques sont évoquées (position en faveur du protocole de Kyoto) en page 4 du document fourni aux experts opposés à la thèse du GIEC, les élèves vont bien au-delà des arguments fournis. Les élèves ont également mis en question l'idée d'une science neutre et indépendante par attribution d'intérêts politiques. Un élève s'interroge sur le paradoxe qui consiste pour un scientifique expert du GIEC à se présenter comme indépendant alors qu'il est sélectionné par son gouvernement. Il pointe également un danger pour les élèves citoyens à suivre les recommandations des experts alors qu'elles impliquent pour lui des choix politiques majeurs qui échappent aux citoyens, bien qu'ayant des conséquences importantes sur leurs modes de vie. Il évoque alors un système dictatorial. Un tel discours nous montre qu'interroger les liens entre science et politique n'est pas hors de portée des élèves et qu'ils sont aptes à considérer le rôle de l'expertise, son usage politique et la place accordée au citoyen. La nature politique de la question du climat a été saisie par les élèves qui ont abordé dans le débat les questions de choix énergétiques, de modes de vie, de croissance économique.

\section{L'autorité de la science et des scientifiques}


30 Par ailleurs, lorsque les élèves discutent des modes d'élaboration des sciences pour contrecarrer l'attribution d'intérêts politiques ou financiers, notre analyse de leurs procédés rhétoriques montre une référence à un travail désintéressé de la part des scientifiques. Ce résultat indique que les élèves perçoivent la science comme une activité de recueil de données, effectuée selon une démarche expérimentale rigoureuse et soignée, fournissant alors des preuves qui sont indiscutables. Les scientifiques sont, dans ce cas, perçus comme objectifs, dévoués, désintéressés. Dans cet esprit, les scientifiques sont vus comme des personnes qui savent et dont on attend qu'ils disent ce qu'ils savent et apportent des solutions. Le citoyen ne se sent pas apte à participer à la décision et la délègue aux experts, comme l'indique l'analyse des procédés rhétoriques relatifs à la position du locuteur. L'analyse des procédés rhétoriques s'appuyant sur l'autorité des experts a également indiqué que, dans leurs jeux de langage, des élèves marquent cette dichotomie experts/citoyens, s'adressant avec déférence et politesse aux experts, déconsidérant les citoyens par l'emploi de qualificatifs dévalorisants.

31 Au cours du débat, plusieurs affirmations appuyées exclusivement sur des données empiriques ont suscité une forme de scepticisme et de remise en cause, ouvrant la porte à des interrogations d'ordre épistémologique, à une remise en question du caractère empirico-réaliste des sciences. De tels propos nous invitent à engager des discussions avec les élèves sur l'élaboration des savoirs scientifiques, leurs validations et plus largement, sur le fonctionnement de la science.

\section{Les savoirs scientifiques mobilisés par les élèves dans le débat}

Les élèves discutent de la question des énergies renouvelables (énergie éolienne, énergie hydroélectrique et hydrolienne, voiture électrique), de leur coût, leur rentabilité, l'entretien de leur matériel, leurs limites en termes de production, leur impact sur le paysage. Leurs échanges à ce propos concernent le souci de préserver l'environnement pour les générations futures, l'actualité politique française, au sein de laquelle l'environnement constitue un enjeu important, la fin du pétrole et le protocole de Kyoto.

I96, EG1: vous citiez tout à l'heure, les élections politiques en France l'année dernière, enfin il y a bientôt un an, les énergies renouvelables telles que les éoliennes, l'énergie solaire. Dernièrement, on a vu qu'il peut y avoir de l'énergie hydrolienne avec des éoliennes, mais qui sont mises sous l'eau, avec les courants, bon tout ce qui est de l'énergie renouvelable, et pas du pétrole, pas des énergies fossiles qui au bout d'un moment vont arriver à épuisement donc on n'en aura plus, donc il faudra bien dans tous les cas trouver une solution et puis des solutions qui respectent en quelque sorte la nature, voilà.

I102, EG1 : je peux le voir arriver sur les éoliennes qui peuvent gâcher le paysage, mais aujourd'hui une éolienne d'accord, c'est grand, ça fait du bruit pour les gens qui habitent en dessous, c'est au niveau de la vision, ce n'est peut-être pas joli [EG1 s'interrompt brièvement pour regarder en direction des citoyens qui chuchotent], mais après vous avez déjà les poteaux électriques qui sont un peu du même style (...)

I103, EG2: [hésitation]

I104, C2 : si je peux me permettre en parlant des éoliennes j'ai rencontré un expert en machinisme qui me disait que les éoliennes c'était une solution bien, mais qu'après à rentabiliser ça...

I105, C3 : ça restait très cher. 
I106, E1 : mais c'est sûr que si on n'a que quelques éoliennes par-ci par-là...

I107, C2 : c'est pas du tout rentable.

I108, E1 : ...c'est sûr que si on n'a que quelques éoliennes par-ci par-là et qu'on veut, par exemple, avec quatre éoliennes, faire vivre un village, ça va être compliqué, mais si on se met à faire en plus de ces éoliennes ... c'est sûr que l'éolienne ce n'est pas la solution.

I109, C2 : non, mais ça se change, vous connaissez le principe de l'éolienne?

I110, E1 : Oui oui

I111, C2 : c'est qu'on ne peut pas garder ça indéfiniment, il faut la réviser. I112, E1 : Je sais très bien. Même les panneaux solaires...

I113, C2 : ça coûte très cher ce change [interruption]

I114, E1 :... même les panneaux solaires au bout de certaines années les cellules solaires ne marchent plus, mais d'un autre côté, comme on était en train de le dire, vous nous posiez la question sur ça... il faut savoir faire quelques concessions pour arriver à un niveau de vie acceptable parce que si les hommes plus tard ont une terre pourrie, comme ce qui peut être prévu, il faut savoir faire quelques efforts, voilà.

I115, C2 : non, mais ce n'est pas à nous de faire les plus gros efforts, c'est à l'État de donner des sous [...]

Les discussions entre élèves portent également sur les changements politicoéconomiques qui peuvent découler de la thèse du GIEC ou y être présentés comme inéluctables dans le contexte d'un changement climatique. Les questions de niveau de vie, de bien-être des citoyens, de l'usage des sciences par les acteurs politiques et du rôle politique des experts sont discutées par les élèves.

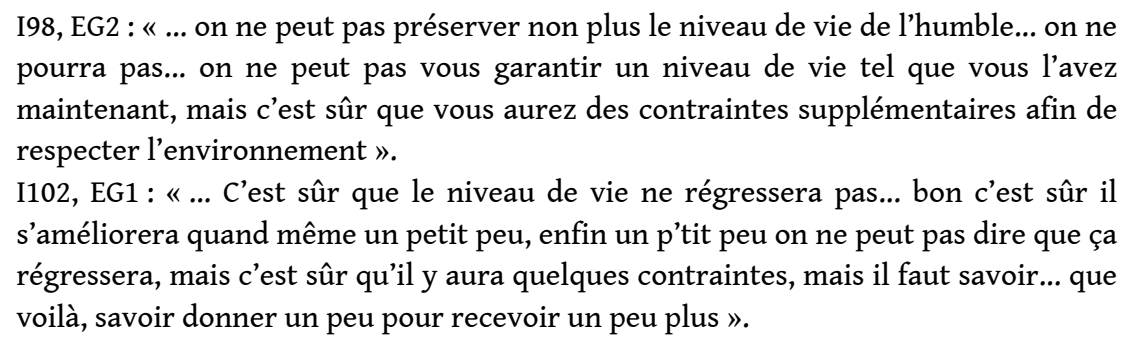

Les élèves, qui jouent le rôle des citoyens, discutent la vision politico-économique présentée par les élèves jouant le rôle des experts du GIEC, refusent une prise en charge individuelle des changements en matière d'énergie et s'en remettent à l'État. Dans le même temps, ils délèguent leur pouvoir aux experts en les sommant en quelque sorte de fournir des solutions et accusent les élèves jouant le rôle des experts opposés à la thèse du réchauffement de ne pas rechercher de solutions.

Les discours des citoyens font ici appel à la preuve.

I221, C2 : « ... c'est à vous de vous lier, de trouver des solutions, pas à nous de dire bon, écoutez une telle et une telle autre».

I234, $\mathrm{C} 1$ : « alors qu'eux ils ont des preuves... ».

Relativement à la vision politico-économique des experts, la question du bien-être du citoyen et du niveau de vie est soulevée par les citoyens, comme l'indiquent les extraits ci-dessous :

I95, C1: « pour conserver notre niveau de vie? ».

I97, C4: «mais seront-elles assez... respectables et en même temps respectant le

bien-être des citoyens?».

Un expert défendant la thèse opposée au réchauffement climatique discute également des conséquences sur le niveau de vie qui découleraient des contraintes imposées pour respecter l'environnement : 
159, E1 : « ce qu'ils veulent vous mettre en place (NDA : l'élève évoque ici les actions des politiciens) c'est justement ça, en parlant d'écologie, et tous vous contraindre dans un mode de vie qui peut-être ne vous satisfera pas ". ches différentes expertises sur les changements climatiques, les élèves mobilisent des informations relevant de données empiriques relatives à l'élévation de température dans le cas d'un réchauffement climatique et aux courbes l'indiquant :

I2, E1 : déjà deux choses : cette augmentation qui est montrée dans leur fameuse courbe est déjà sur un temps trop court. Sur le dernier siècle, on a pu avoir des relevés précis grâce au thermomètre et on a vu une augmentation de $0,6^{\circ}$ sauf que, au début, on était avec nos pauvres petits thermomètres à mercure et maintenant nous sommes avec des thermomètres électroniques. Après, par rapport au siècle, les mille ans qui ont été faits avant [NDA : les modélisations des climats du passé] ils ont fait par rapport aux carottes de glace, aux arbres [NDA : les données utilisées dans les modèles]. En regroupant ces données, ils ont réussi à élaborer cette courbe, sauf que les scientifiques de terrain, qui eux n'ont rien à prouver juste à constater, ont expliqué qu'il y avait certaines dynamiques des glaces qui avaient changé en plusieurs siècles et que, par exemple, il fallait plus se fier pendant les quelques millénaires, le siècle avant dernier c'était plus sur les bulles de $\mathrm{CO} 2$ qu'il fallait faire parce qu'elles étaient trompées ou faussées par... le soleil ou «ché " plus quoi le soleil voilà » [NDA : l'élève évoque la théorie des changements climatiques qui s'appuie sur la distance et l'inclinaison Terre-Soleil]. 
I241, E1 : on est en train de dire que leurs courbes ne sont pas valables.

43 La dynamique des glaces et une référence à l'influence du soleil sont également présentes dans le discours de cet élève jouant le rôle d'un expert opposé au réchauffement climatique.

\section{Les élèves et la dimension politique des controverses sur le climat}

L'enjeu majeur de la controverse autour du réchauffement climatique et la nature politique de la question du climat sont discutés par les élèves. Ce résultat nous apparaît remarquable dans la mesure où nombre de discours médiatiques sur la question se centrent sur les conséquences d'un réchauffement climatique (inondations, fonte des glaces, réduction de la biodiversité, réfugiés...) et sur leur dimension spectaculaire ou dramatique, ou encore questionnent les causes d'un tel réchauffement (rôle anthropique, alternance naturelle de périodes glaciaires/interglaciaires, etc.). Ici, les élèves n'ont pas abordé ce registre dramatico-médiatique mais ont pointé le lien entre climat et énergie et soulevé des questions sur la prise en charge politique des problèmes. Le fait que l'élaboration de solutions soit confiée à certains experts, dont des liens avec la sphère politique sont soulevés par les élèves, est perçu, pour certains d'entre eux, comme un risque pour la démocratie, dans la mesure où le pouvoir de décider reviendrait aux seuls experts. D'autres élèves, jouant le rôle de citoyens, sollicitent des preuves scientifiques, demandent l'accord entre chercheurs et délèguent l'élaboration de solutions à ces derniers, dépeignant une vision technocratique de l'expertise.

Les relations sciences - politique sont ainsi discutées par les élèves, ce qui concourt à leur formation citoyenne. Ces échanges les encouragent à identifier des situations qui leur permettraient de prendre leur pouvoir et de ne pas se soumettre aux discours des autres, à porter des jugements autonomes, à se sentir légitimes, à participer aux décisions, autrement dit, aptes à participer à la configuration de leur monde.

\section{La scolarisation de questions politiques}

La recherche empirique que nous venons de présenter nous a - suite à sa réalisation conduite à nous interroger sur la scolarisation de questions politiques. Étant donné le caractère pionnier de notre étude, nous avons engagé une confrontation entre les résultats de notre recherche, inscrite dans le champ de la didactique des sciences, à ceux issus de recherches menées en didactique des sciences économiques et sociales. Une première tentative de confrontation de la prise en charge de questions politiques dans l'enseignement secondaire français, de sciences économiques et sociales et de sciences de la matière a donc été menée (Chatel et Albe, 2010). Cette tentative s'appuie sur deux idées majeures. D'une part, nous pensons que la politique concerne l'organisation de la vie de la cité et que, par conséquent, elle interpelle les acteurs scolaires, elle nous interpelle tous. D'autre part, nous avons considéré les critiques, positions de refus ou de mises en garde d'enseignants au nom de la nécessité d'installer et de maintenir en classe un climat serein, loin de "conflits d'opinions » ou d'oppositions partisanes, potentiellement générateurs de tensions ou de violence. C'est la tension entre acuité politique des questions d'intérêt social large et nécessité de sérénité en classe qui a constitué le point de départ de notre travail. La confrontation 
que nous avons engagée sur l'intégration des questions politiques dans l'enseignement des sciences et des sciences économiques et sociales nous a ensuite conduites à explorer les débats sur la légitimité sociale de curricula traitant de ces questions. Des considérations épistémologiques y jouent un rôle majeur.

En classe, un enjeu consiste - dans les deux disciplines d'enseignement -, à faire passer les élèves de l'indifférence, de l'importation d'opinions personnelles ou parentales ou des discours médiatiques, sans mise en distance ou confrontation violente à une étude documentée des questions intégrant des éléments scientifiques. Mais précisons tout de suite que l'étude scolaire de problèmes sociaux contemporains par le recours aux sciences telle que nous l'avons envisagée ne s'apparente pas à l'approche qui revient à refuser la querelle politique pour ne retenir que les questions scientifiques et à placer les élèves en situation de recherche d'une vérité unique. Cette démarche est, selon nous, problématique pour plusieurs raisons. Sur un plan pédagogique, si l'on souhaite traiter de questions qui ont du sens pour les élèves, dans leur vie d'aujourd'hui ou de demain, rabattre l'enjeu politique des questions sur un traitement scientifique nous apparaît comme peu tenable pour des raisons éthiques. L'entrée "politique " nous apparaissant dans ce cas comme une " accroche » motivante et mensongère, qui - pour le dire brutalement - peut s'apparenter à une invitation sous la forme "viens au cours où l'on parle de choses qui t'intéressent et te concernent, et où on discutera de politique » pour ensuite muer la proposition en «faisons des maths ». La question des élèves qui se fait parfois insistante - «quel sens cela a-t-il pour moi ?» - peut trouver ici une légitimité criante. Soulignons d'ailleurs que les élèves peuvent en quelque sorte « remettre du politique » là où les enseignants chercheraient à l'éviter. En outre, sur un plan épistémologique, cette transformation en traitement scientifique renvoie à une image mythique de la science, à la recherche d'une vérité unique et nous y reviendrons plus loin.

Un travail spécifique et guidé sur la documentation nous apparait ici comme une voie potentiellement fructueuse. Elle peut conduire à une éducation aux médias et à la discussion en classe des questions de l'autorité et du pouvoir. L'analyse de dossiers documentaires constitue en sciences économiques et sociales une pratique pédagogique ordinaire, alors qu'en sciences physiques, il s'agit d'une pratique peu coutumière, rompant avec la forme scolaire traditionnelle centrée sur l'étude de phénomènes, concepts, lois, théories, mis à l'épreuve expérimentale. Toutefois, comme nous l'avons présenté plus haut à propos d'une recherche collaborative sur les changements climatiques, de telles pratiques d'analyse de documents se développent le plus souvent en interdisciplinarité avec un documentaliste, un enseignant de philosophie et/ou des chercheurs en éducation. Bien qu'exceptionnel, ce type de traitement de questions politiques en classe de sciences est possible, comme la recherche empirique présentée plus haut l'indique. Les élèves sont aptes, et cela, même si ce n'était pas objet ou enjeu de l'enseignement, à cerner les questions scientifiques, leur dimension politique, et à discuter des liens entre sciences, expertises et politique. Cela implique pour eux de reconnaître les approches différentes des questions entre scientifiques et experts, de percevoir le rôle des acteurs politiques et les enjeux économiques imbriqués dans les sciences, les expertises et leur développement, et de discuter de la façon dont des expertises se construisent et de leurs usages politiques.

Il s'agit de rendre les élèves plus vigilants et moins dominés par l'autorité de la science et des experts, de les aider à se ressaisir à terme des questions qui doivent être prises 
en charge par les citoyens. En sciences économiques et sociales, cet objectif est satisfait lorsque l'on donne aux élèves des outils conceptuels et théoriques, certes schématiques, pour bien appréhender cette ressaisie des questions politiques. En sciences physiques, le bilan relatif à cet «empowerment " visé dans les recherches en didactique des sciences est nuancé. D'une part, l'étude d'expertises scientifiques contradictoires sur des controverses socialement vives, la pratique de la critique et le développement d'une expertise outillée sur le plan épistémologique peuvent être considérés comme favorables à cette ressaisie du politique. D'autre part, la remise en cause du mythe de l'autorité de la science pourvoyeuse d'une vérité unique, permettant d'orienter directement l'action politique, est perturbante et peut laisser les élèves perplexes. Nous percevons un risque si cela conduisait à croire que « rien ne vaut » et/ ou à se détourner de la réflexion politique, au sens de configuration du futur et de la vie de la cité.

Par ailleurs, quant à la légitimité d'intégration de questions politiques dans les curriculums, existent des désaccords entre enseignants de sciences économiques et sociales et des réticences à enseigner "des questions de société où la science est impliquée » chez les enseignants de sciences (Levinson et Turner, 2001 ; Sadler et al., 2006 ; Albe et Ruel, 2008).

51 Un examen des considérations épistémologiques en jeu dans les débats entre professeurs nous a conduites à identifier chez eux une référence à un modèle particulier de scientificité des sciences de la nature, et à une approche à saveur bachelardienne et/ou poppérienne, où la science est vue comme une réponse à une question par recours à l'expérimentation et à la réfutation des hypothèses. La science est perçue comme méthode pour parvenir à une vérité unique qui clôturerait les controverses. Une telle image peut fonctionner comme un idéal, mais ne permet pas de rendre compte de la nature et des pratiques effectives de sciences. Les savoirs scientifiques se présentent plutôt comme des modèles divers concernant le projet d'élaboration de connaissances. Une diversité de positions peut également se constituer lorsque sont en jeu les conceptions de l'humain et de son avenir, par exemple à propos du clonage ou des nanotechnologies lorsque se pose la question du transhumanisme ou de la surveillance des individus. En sciences économiques, ces considérations sur l'humain et son avenir, ainsi que sur les conceptualisations de l'être humain, de l'action humaine et des relations sociales, sont au cœur de la diversité des approches. En sciences de la matière et du vivant, la pluralité des modèles et des modes de validation des connaissances est au centre de la construction des savoirs, et la controverse n'est pas à éviter, mais bien au contraire « constitue le milieu natal de la construction des faits " (Stengers, 1997, p. 85-86). L'unicité d'un modèle de scientificité fait problème. Penser la scientificité des sciences économiques et sociales en référence à un idéal des sciences de la nature éloigné de leur histoire, de leurs pratiques et savoirs reviendrait, d'une part à nier la nature politique des sciences économiques tant dans leur objet que dans leurs principes (Chatel et Albe, 2010), et d'autre part à continuer de faire vivre une dichotomie science/politique ou une recherche du vrai/du juste à propos des sciences. Si l'on s'intéresse aux pratiques scientifiques telles qu'elles se font, les approches des sciences en termes de ruptures, contre les pensées premières pour Bachelard (1980) et via les réfutations chez Popper $(1973,1985)$, sont remises en question. Si ces approches peuvent résister pour l'étude de la science déjà faite, elles montrent des signes d'obsolescence pour l'étude des sciences en développement, et en particulier en ce qui concerne les controverses scientifiques contemporaines. Il est alors nécessaire de 
rouvrir la question épistémologique afin de repenser les sciences et leur socialité. Le champ des études sociales sur les sciences (ou science studies) est ici riche d'enseignements pour s'engager dans une refondation des sciences à l'école.

Au contraire, naturaliser dans le discours des représentations situées non reconnues comme telles pour faire apprendre un vrai unique et indiscutable nous semble relever d'un tour de passe-passe intellectuel. L'enjeu épistémologique rejoint ici l'enjeu socioéducatif déjà formulé. Si, dans l'enseignement, les savoirs scientifiques sont enseignés comme des réalités à l'œuvre et que n'y est pas abordée la question de leur pluralité et de leur pertinence dans la situation étudiée, ni raisonné le choix d'un modèle ou des modes de découpage ou de construction des objets, l'éducation des élèves nous apparaît destinée à leur insertion sans questionnement dans un monde prétendu naturel (le monde tel qu'il est). Ici affleure l'essentielle question de l'enjeu sociopolitique de l'éducation. Se pose la question de la formation d'un être complet et pas seulement d'un être cognitif, en dehors du monde ou avec un rapport au monde, qui se développe en dehors de l'école. S'agit-il de présenter un monde naturalisé comme unique voie pour l'avenir, comme une " nécessité », ou plutôt de donner à cette représentation le statut d'une "possibilité » parmi d'autres? S'agit-il de viser une insertion dans un social indiscutable ou plutôt une émancipation des individus et des sociétés? L'enjeu socio-éducatif majeur consiste-t-il à favoriser une participation à une (re)configuration du monde?

\section{BIBLIOGRAPHIE}

Albe, V. (2005). Un jeu de rôle sur une controverse socio-scientifique actuelle : Une stratégie pour favoriser la problématisation? Aster, 40, 67-94.

Albe, V. (2007). Des controverses scientifiques socialement vives en éducation aux sciences. État des recherches et Perspectives. Mémoire de synthèse pour l'Habilitation à diriger des recherches. Université Lyon 2.

Albe, V. (2008). Pour une éducation aux sciences citoyenne : Une analyse sociale et épistémologique des controverses sur les changements climatiques. Aster, 46, 45-70.

Albe, V. (2009). Enseigner des controverses. Rennes : Presses Universitaires de Rennes.

Albe, V. et Gombert, M.-J. (2010). Intégration scolaire d'une controverse socioscientifique contemporaine : savoirs et pratiques d'élèves pour appréhender les savoirs et pratiques de scientifiques. Recherches en didactiques des sciences et des technologies, 2 (sous presse).

Albe, V. et Ruel, F. (2008). Rapport au savoir scientifique et enseignement de controverses scientifiques socialement vives. Communication présentée au colloque Rapport au savoir scientifique, éducation aux sciences et à l'environnement du 76e Congrès de l'ACFAS, Québec, 7-8 mai 2008.

Bachelard, G. (1980). La formation de l'esprit scientifique : contribution à une psychanalyse de la connaissance objective. Paris : Vrin. 
Bader, B. (2003). Interprétation d'une controverse scientifique : stratégies argumentatives d'adolescentes et d'adolescents québécois. Revue canadienne de l'enseignement des sciences, des mathématiques et des technologies, 3, 231-250.

Chatel, E. et Albe, V. (2010). La scolarisation de questions politiques en sciences économiques et sociales et en sciences physiques. Communication présentée au Séminaire Curricula et confrontations internationales, organisé par le laboratoire Sciences techniques éducation formation (STEF) de l'École normale supérieure de Cachan, 19 mars 2010.

Dawes, L. (2004). Talk and learning in classroom science. International Journal of Science Education, 26, 677-695.

Driver, R., Leach, J., Millar, R. et Scott, P. (1996). Young people's image of science. Buckingham : Open University Press.

Edwards, D. (1997). Discourse and cognition. London : Sage.

Gombert, M.-J. (2008). Contribution à une éducation scientifique au travers d'un débat favorisant des échanges non affaiblissants entre élèves sur la question socioscientifique du réchauffement climatique. Master 2 Recherche, Université de Lyon 1 et Université Montpellier 2.

Kolstø, S. D. (2001). 'To trust or not to trust, ...'-pupils' ways of judging information encountered in a socio-scientific issue. International Journal of Science Education, 23, 877-901.

Larochelle, M. et Désautels, J. (2006). L'éducation aux sciences et le croisement des expertises. Dans Legardez, A. et Simonneaux, L., L'école à l'épreuve de l'actualité. Enseigner les questions vives (p. 61-77). Paris : ESF.

Legardez, A. (1999). Voies de recherche en didactique des sciences économiques, sociales et de gestion: l'exemple des Sciences Economiques et Sociales dans l'enseignement secondaire français. Mémoire de synthèse pour l'habilitation à diriger des recherches. Université de Provence.

Legardez, A. (2006). Enseigner des questions socialement vives. Quelques points de repères. Dans Legardez, A. et Simonneaux, L., L'école à l'épreuve de l'actualité. Enseigner les questions vives (p. 19-31). Paris : ESF.

Legardez, A. et Alpe, Y. (2001). La construction des objets d'enseignements scolaires sur des questions socialement vives : problématisation, stratégies didactiques et circulations des savoirs. In Actes du quatrième Congrès AECSE, Actualité de la recherche en éducation et formation. Paris : AECSE.

Legardez, A. et Simonneaux, L. (2006). L'école à l'épreuve de l'actualité. Enseigner les questions vives. Paris : ESF.

Levinson, R. et Turner, S. (2001). The teaching of social and ethical issues in the school curriculum, arising from developments in biomedical research : a research study of teachers. London : Final Report to The Wellcome Trust.

Mercer, N. (1996). The Guided Construction of Knowledge : Talk Amongst Teachers and Learners. Clevedon : Multilingual Matters.

Popper, K. (1973). La logique de la découverte scientifique. Paris : Payot. Popper, K. (1985). Conjectures et réfutations. La croissance du savoir scientifique. Paris : Payot. Potter, J. (1997). Representing reality. Discourse, rhetoric and social construction. London : Sage.

Rosenberg, M. B. (1999). Les mots sont des fenêtres (où bien ce sont des murs). Initiation à la communication non violente. Paris : La Découverte et Syros. 
Sadler, T. D. (2004). Informal reasoning regarding socioscientific issues : a critical review of research. Journal of Research in Science Teaching, 41, 513-536.

Sadler, T. D. (2009). Situated learning in science education : socio-scientific issues as contexts for practice. Studies in Science Education, 45, 1-42.

Sadler, T. D., Amirshokoohi, A., Kazempour, M. et Allspaw, K. M. (2006). Socioscience and ethics in science classrooms : Teacher perspectives and strategies. Journal of Research in Science Teaching, 43, 353-376.

Stengers, I. (1997). Sciences et Pouvoirs. Faut-il en avoir peur ? Labor : Bruxelles.

\section{NOTES}

1. Virginie Albe est professeure de didactique des sciences et des techniques au laboratoire STEF de l'École normale supérieure de Cachan. Marie-José Gombert est enseignante de sciences physiques au lycée agricole de Rodez (France) et titulaire d'un Master 2 Recherche en histoire, philosophie et didactique des sciences des Universités Claude Bernard Lyon 1, Montpellier 2 et ENS Lyon.

2. Le film «Une vérité qui dérange » est la version française du documentaire américain intitulé « An Inconvenient Truth », réalisé en 2006 par Davis Guggenheim. Il s'agit d'un plaidoyer de l'ancien Vice-président des États-Unis, Al Gore, pour persuader ses concitoyens de l'urgente nécessité de réagir à la crise climatique établie par les experts internationaux du climat (GIEC). Si rien n'était fait, le dérèglement majeur du système climatique entraînerait des catastrophes d'une ampleur sans précédent (perturbations météorologiques extrêmes, inondations, longues périodes de sécheresse, vagues de chaleur meurtrières, etc.).

3. Les élèves ont pratiqué cela dès le début de l'année scolaire avec, par exemple, l'analyse d'un film sur les dons et trafics d'organes pour aborder les questions d'éthique, et celle d'un film présentant le cas d'un homme atteint de ce que la médecine appelle la "locked-in syndrome » pour aborder la question de la conscience.

4. Initialement, il était prévu un nombre de huit citoyens, mais un élève était absent ce jour-là.

5. CNV1 et $\mathrm{CNV} 2$ désignent les deux élèves qui sont intervenus au cours du débat comme modératrices en communication non violente; $\mathrm{C} 1$ à $\mathrm{C}$, les élèves-citoyens ; $\mathrm{EG1}$ et $\mathrm{EG} 2$, les élèves chargés de présenter la thèse des experts du GIEC; E1 et E2, les élèves chargés de présenter la thèse d'experts opposés au réchauffement climatique d'origine anthropique.

6. Une présentation détaillée des analyses des procédés rhétoriques développés par les élèves a fait l'objet d'un article à paraître dans la revue Recherches en didactiques des sciences et des technologies, 2 (Albe et Gombert, 2010).

\section{RÉSUMÉS}

Dans cet article, nous examinons la question de l'intégration scolaire de controverses socioscientifiques qui mobilisent et divisent scientifiques, experts, citoyens, et qui participent à la configuration de la société. Leur nature politique est peu examinée comme telle dans les recherches en éducation et en classe. Nous présentons une recherche empirique menée en 
collaboration interdisciplinaire avec des enseignants de sciences et de philosophie et portant sur l'étude, par des élèves de l'enseignement secondaire technologique français, des controverses sur les changements climatiques. Les résultats indiquent que les élèves s'intéressent à la dimension politique des changements climatiques, aux controverses qu'ils entraînent et aux expertises développées à leur propos. Nous discutons ensuite, d'un point de vue curriculaire, des potentialités de prise en charge de questions politiques en classe de sciences.

In this article, we examine the integration, at school, of socioscientific controversies mobilizing and dividing scientifics, experts, citizens and participating in society's configuration. The political nature of these questions is not often considered in classrooms and received so far little attention within the education research field. We present an empirical research, undertaken in an interdisciplinary collaborative way with science and philosophy teachers, in which we analyzed situations where students discussed about climate changes controversies. Results indicate that students are interested in the political dimension of climate changes, in the controversies they induce and in the expertises that were developed about the phenomenon. We finally discuss, from a curricular perspective, the potentialities of broaching political issues in science classes.

\section{AUTEUR}

\section{VIRGINIE ALBE}

Professeure de didactique des sciences et des techniques au laboratoire Sciences techniques éducation formation (STEF) de l'École normale supérieure de Cachan, ses recherches portent sur les orientations contemporaines de l'éducation scientifique et technique qui tiennent compte des mutations des pratiques technoscientifiques et des enjeux sociétaux. L'enjeu socio-éducatif de telles recherches consiste à développer chez les jeunes une compréhension avertie de l'imbrication des questions de sciences, de techniques et de société, un usage raisonné et critique des expertises scientifiques et une participation démocratique aux débats publics. Elle est l'auteure de l'ouvrage « Enseigner des controverses », paru aux Presses Universitaires de Rennes en 2009. 